\title{
Prevalence of different HIV-1 subtypes in sexual transmission in China: a systematic review and meta-analysis
}

\author{
R. $\mathrm{YUAN}^{1} \dagger, \mathrm{H} \cdot \mathrm{CHENG}^{2} \dagger$, L.-S. $\mathrm{CHEN}^{1}, \mathrm{X} \cdot \mathrm{ZHANG}^{2}$ AND B. WANG ${ }^{1 *}$ \\ ${ }^{1}$ Department of Epidemiology and Health Statistics, School of Public Health, Southeast University, Nanjing, \\ China \\ ${ }^{2}$ Wuxi Center for Disease Control and Prevention, Wuxi, Jiangsu, China
}

Received 11 September 2015; Final revision 7 January 2016; Accepted 20 January 2016; first published online 19 February 2016

\section{SUMMARY}

Sexual transmission has become the primary route of HIV transmission in China. Therefore, a comprehensive overview of HIV-1 subtype distribution is necessary for the prevention and control of the HIV epidemic. The present study aimed to provide a comprehensive prevalence estimate of different HIV-1 subtypes in sexual transmission in China. We conducted a systematic literature review for studies of HIV-1 subtypes in English and Chinese through several databases. Eligible articles were screened and selected by two authors independently. Random-effects model were applied to calculate the pooled prevalence of different HIV-1 subtypes, and subgroup analyses examined prevalence estimates across time, locations, and populations. A total of 130 eligible studies were identified, including 18752 successfully genotyped samples. The pooled prevalence of CRF01_AE, subtype B, CRF07_BC, CRF08_BC, and subtype C were 44.54\% (95\% CI 40.81-48.30), 18.31\% (95\% CI 14.71-22.17), 16.45\% (95\% CI 13.82-19.25), $2 \cdot 55 \%$ (95\% CI 1.56-3.73), 0.37\% (95\% CI $0 \cdot 11-0 \cdot 72$ ), respectively. The prevalence of subtype $\mathrm{B}$ in sexual transmission decreased, while the prevalence of CRF01_AE and CRF07_BC in sexual transmission, and CRF08_BC in heterosexual transmission increased. There is significant variation in HIV-1 subtype distribution between regions. The distribution of HIV-1 subtypes and circulating recombinant forms have changed significantly. The high genetic variability of HIV-1 poses a significant challenge for disease control and surveillance in China.

Key words: China, HIV-1, sexual transmission, subtype.

\section{INTRODUCTION}

The HIV and AIDS epidemic in China remains very serious. Incidence of HIV/AIDS continues to increase annually, from 21700 in 2003 to 90100 in 2013 [1]. In 2011, an estimate of 780000 (range 620 000-940 000) individuals had HIV/AIDS in China [2]. By the end of July 2015, 554602 people were reported to be infected

\footnotetext{
* Author for correspondence: Professor B. Wang, Department of Epidemiology and Health Statistics, School of Public Health, Southeast University, Nanjing 210009, China. (Email: wangbeilxb@163.com)

$\dagger$ These authors contributed equally to this work.
}

with HIV/AIDS and 171186 died from AIDS-related illnesses [3]. In the last 10 years, the main drivers of the HIV epidemic in China have shifted, from primarily blood-borne spreading to sexual contact. Of the new cases diagnosed annually, the percentage of sexually transmitted cases increased from $33.1 \%$ in 2006 to $90.8 \%$ in 2013 , with the male homosexual transmission rate increasing from $2.5 \%$ in 2006 to $21.4 \%$ in 2013 [4].

HIV is characterized by a high genetic diversity and extensive heterogeneity. This characteristic is due to multiple factors including multiple zoonotic transmissions into human populations, high rates of viral 
evolution, and recombination. HIV has two major types, HIV-1 and HIV-2, which are further divided into groups, subtypes, and recombinant forms [5]. Globally, over $90 \%$ of the HIV infections belong to HIV-1 group $M$ viruses, which have been further classified into nine subtypes (A-D, F-H, J, K) and more than 72 circulating recombinant forms (CRFs). Additionally, there are many unique recombinant forms (URFs) that are globally distributed [6, 7]. The spread of HIV-1 variants has implications for disease diagnosis, treatment, and vaccine development [8].

In China, the distribution of HIV-1 subtypes is complex and diverse. Previous studies have shown that HIV-1 prevalent strains include at least 30 subtypes, including nine subtypes and more than 21 CRFs [6, 8-10]. Of these, CRF01_AE, CRF07_BC, CRF08_BC, and subtype B are the predominant circulating HIV-1 subtypes [9-11]. This diversity presents a serious challenge for HIV prevention and treatment strategies. However, HIV strains often show specific associations with geographical regions and modes of transmission [12, 13]. Nevertheless, HIV-1 subtypes are possibly related to disease progression and drug resistance $[14,15]$. Through surveillance of the dynamic changes of HIV-1 genetic variability, we can track the source of infection and develop public health strategies in a timely manner in order to effectively prevent the virus spreading. This will also benefit HIV-1 diagnosis, treatment, and vaccine development [16]. Currently, sexual transmission has become the primary mode of HIV-1 spread in China, but little is known about HIV-1 subtype distribution in sexual transmission and this should be the primary focus.

The main objective of this review was to provide a comprehensive overview and pooled prevalence estimate of different HIV-1 subtypes in sexual transmission in China. In addition, we also analysed the temporal and geographical variation from the data to provide more detailed information for the effective prevention and control of HIV-1 in China.

\section{METHOD}

We followed the Preferred Reporting Items for Systematic Reviews and Meta-Analyses (PRISMA) guidelines to perform and report this meta-analysis [17].

\section{Search strategy}

We conducted a literature search in English and Chinese using the following databases: PubMed,
Web of Science, China National Knowledge Infrastructure (CNKI), Chinese Biomedical Literature Database (CBM), and Wanfang, from inception to 10 December 2015. Using the following search items: (HIV OR human immunodeficiency virus OR AIDS OR acquired immunodeficiency syndrome) AND (genotype OR subtype OR molecular epidemiology) AND China. Additionally, we reviewed the reference lists of the retrieved articles for additional relevant studies.

\section{Selection criteria}

Two authors (R.Y. and H.C.) independently assessed the eligibility of the articles, and a third reviewer (B.W.) supervised this process when opinions were divergent. The following criteria were applied: (1) studies based on HIV-1 subtypes in China; (2) modes of transmission must contain sexual transmission, such as bisexual, heterosexual (HST), or men who have sex with men (MSM) transmission; (3) provide primary data of the frequency of each subtype; (4) provide available information on study time and location; and (5) sample size of more than 10. Exclusion criteria included studies on a single specific subtype or the assessment of articles on detection techniques. To avoid duplication, if the same study data were reported repeatedly, the one that provided more detailed information and/or a larger sample size was chosen.

\section{Data extraction}

According to the selection criteria, two authors (R.Y. and H.C.) independently extracted study data, including first-named author, year of publication, study period, study locations, study populations (or modes of transmission), study method of classification of HIV-1 subtypes, sample size, number of successfully genotyped samples and the frequency of each subtype. Any differences were resolved by consensus. As for the method of HIV-1 classification, sequence-based typing was viewed as the 'gold standard' [18]. In particular, manual phylogenetic analysis of the pol, gag and env regions was widely applied to HIV subtyping in China because automated subtyping tools do not provide accurate subtype assignment for complex strains such as CRF07_BC, CRF08_BC, etc. Therefore, all of the included studies determined HIV-1 subtypes based on pol, gag and/or env gene amplification and sequencing. 


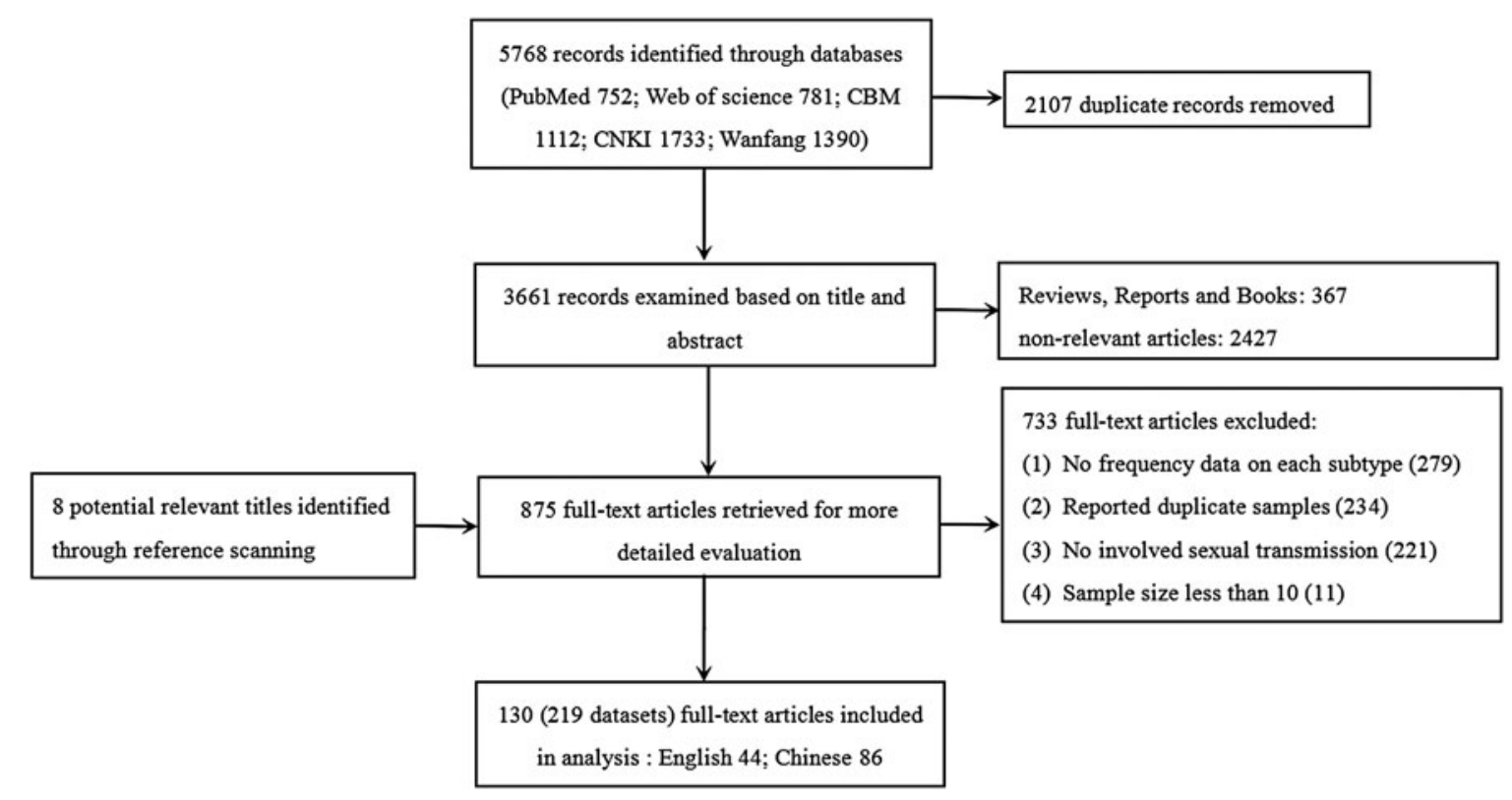

Fig. 1. Summary of literature search and selection procedure.

\section{Statistical analysis}

The Freeman-Tukey double arcsine transformation of proportions was used to estimate the pooled proportion of different HIV-1 subtypes [19, 20]. Heterogeneity between the studies was examined using Cochran's $Q$ statistic and the $I^{2}$ statistic [21, 22]. Depending on the heterogeneity, A mixed-effects model was chosen to calculate the pooled point estimate: random-effects models were selected when the $P$ value of Cochran's $Q$ statistic was $<0 \cdot 1$ or $I^{2}$ was $>50 \%$, or fixed-effects models were used. Subgroup analyses were conducted based on the time period, study location, and study population to further assess heterogeneity. The funnel plot and Egger's test were used to assess publication bias, which found no evidence of bias (Supplementary Fig. S1). All analyses were performed using R software (version 3.2.1) [23] and SAS (version 9.3, SAS Institute Inc., USA).

\section{RESULTS}

\section{Study selection and characteristics}

Figure 1 summarizes the literature search results and selection procedure. Of the 5768 abstracts (4234 in Chinese, 1533 in English) initially identified, 130 unique articles were finally included. Most articles were identified through database searching, and only three (out of eight potentially relevant titles) additional eligible articles were identified through bibliography scanning. The 130 studies were divided into 219 datasets based on different study times, locations, or populations. The study period ranged from 1988 to 2014 . Five studies were from the national survey of HIV-1 subtypes. Of the remainder, study sites were distributed throughout 28 different provinces or regions. Eventually, 18752 successfully genotyped samples were included in the analysis which comprised 6759 heterosexuals, 10516 MSM, 198 bisexuals, and 1279 uncertain unprotected sexual contact individuals. More detailed information is included in Supplementary Table S1.

\section{Proportion of different HIV-1 subtypes}

The prevalent HIV-1 strains in sexual transmission were commonly CRF01_AE, CRF07_BC, CRF08_BC, and subtype B (including US-European B and Thailand B). Comprehensive analysis of the pooled proportion of different HIV-1 subtypes to CRF01_AE, CRF07_BC, CRF08_BC, B/B', C, and others was $44 \cdot 54 \%$ [ $95 \%$ confidence interval (CI) 40.81-48.30], 16.45\% (95\% CI 13.8219.25), $2 \cdot 55 \%$ (95\% CI $1 \cdot 56-3 \cdot 73), 18 \cdot 31 \%(95 \%$ CI $14 \cdot 71-22 \cdot 17), 0 \cdot 37 \%(95 \%$ CI $0 \cdot 11-0 \cdot 72)$, and $2 \cdot 01 \%$ (95\% CI $1 \cdot 36-2 \cdot 75)$, respectively.

\section{Subgroup analysis}

As shown in Table 1, the proportion of different HIV-1 subtypes was further explored within identifiable 
Table 1. Pooled prevalence of different HIV-1 subtypes stratified by time period, study region and risk group

\begin{tabular}{|c|c|c|c|c|c|c|c|c|c|c|}
\hline \multirow[b]{2}{*}{ Category } & \multirow[b]{2}{*}{$N$} & \multicolumn{3}{|l|}{ CRF01_AE } & \multicolumn{3}{|l|}{ CRF07_BC } & \multicolumn{3}{|l|}{ CRF08_BC } \\
\hline & & $\begin{array}{l}\text { Proportion } \\
\%(95 \% \text { CI })\end{array}$ & $P^{*}, I^{2}$ & $P \dagger$ & $\begin{array}{l}\text { Proportion } \\
\%(95 \% \text { CI })\end{array}$ & $P^{*}, I^{2}$ & $P \dagger$ & $\begin{array}{l}\text { Proportion } \\
\%(95 \% \text { CI })\end{array}$ & $P^{*}, I^{2}$ & $P \dagger$ \\
\hline Region & & & & $0 \cdot 001$ & & & $<0 \cdot 01$ & & & $<0 \cdot 01$ \\
\hline Northeastern & 22 & $57 \cdot 80(45 \cdot 74-69 \cdot 44)$ & $<0 \cdot 01,94 \cdot 0 \%$ & & $4 \cdot 63(2 \cdot 57-7 \cdot 13)$ & $<0 \cdot 01,58 \cdot 2 \%$ & & $0 \cdot 00(0 \cdot 00-0 \cdot 00)$ & $0 \cdot 757,0.0 \%$ & \\
\hline Eastern & 115 & $48 \cdot 95(44 \cdot 17-53 \cdot 74)$ & $<0 \cdot 01,95 \cdot 7 \%$ & & $12 \cdot 47(9 \cdot 84-15 \cdot 35)$ & $<0 \cdot 01,93 \cdot 8 \%$ & & $1 \cdot 33(0 \cdot 64-2 \cdot 19)$ & $<0 \cdot 01,85 \cdot 3 \%$ & \\
\hline Central & 27 & $34 \cdot 41(24 \cdot 75-44 \cdot 72)$ & $<0 \cdot 01,94 \cdot 2 \%$ & & $19 \cdot 52(11 \cdot 78-28 \cdot 51)$ & $<0 \cdot 01,94 \cdot 1 \%$ & & $0 \cdot 14(0 \cdot 00-0 \cdot 84)$ & $0 \cdot 004,46 \cdot 8 \%$ & \\
\hline Northwestern & 7 & $22 \cdot 68(4 \cdot 31-48 \cdot 76)$ & $<0 \cdot 01,96 \cdot 7 \%$ & & $65 \cdot 89(35 \cdot 98-90 \cdot 41)$ & $<0 \cdot 01,97 \cdot 3 \%$ & & $0 \cdot 00(0 \cdot 00-0 \cdot 39)$ & $0 \cdot 153,36 \cdot 1 \%$ & \\
\hline Southwestern & 45 & $36 \cdot 16(28 \cdot 16-44 \cdot 55)$ & $<0 \cdot 01,96 \cdot 1 \%$ & & $25 \cdot 94(18 \cdot 20-34 \cdot 46)$ & $<0 \cdot 01,96 \cdot 7 \%$ & & $13 \cdot 93(7 \cdot 93-21 \cdot 13)$ & $<0 \cdot 01,96 \cdot 7 \%$ & \\
\hline Time period§ & & & & $0 \cdot 001$ & & & $<0 \cdot 01$ & & & $0 \cdot 164$ \\
\hline Before 2000 & 9 & $29 \cdot 11(15 \cdot 37-44 \cdot 92)$ & $<0 \cdot 01,95 \cdot 5 \%$ & & $0 \cdot 59(0 \cdot 00-3 \cdot 92)$ & $<0 \cdot 01,85 \cdot 3 \%$ & & $0 \cdot 44(0 \cdot 00-3 \cdot 34)$ & $<0 \cdot 01,82 \cdot 8 \%$ & \\
\hline 2001-2004 & 14 & $28 \cdot 73(17 \cdot 30-41 \cdot 61)$ & $<0 \cdot 01,89 \cdot 8 \%$ & & $6 \cdot 44(1 \cdot 74-13 \cdot 18)$ & $<0 \cdot 01,84 \cdot 2 \%$ & & $6 \cdot 72(1 \cdot 39-14 \cdot 72)$ & $<0 \cdot 01,88 \cdot 1 \%$ & \\
\hline 2005-2007 & 34 & $33 \cdot 30(22 \cdot 76-44 \cdot 69)$ & $<0 \cdot 01,97 \cdot 5 \%$ & & $10 \cdot 53(6 \cdot 04-15 \cdot 93)$ & $<0 \cdot 01,94 \cdot 0 \%$ & & $4 \cdot 67(1 \cdot 60-8 \cdot 86)$ & $<0 \cdot 01,94 \cdot 1 \%$ & \\
\hline 2008-2010 & 86 & $49 \cdot 92(43 \cdot 96-55 \cdot 88)$ & $<0 \cdot 01,95 \cdot 6 \%$ & & $15 \cdot 04(11 \cdot 50-18 \cdot 92)$ & $<0 \cdot 01,93 \cdot 8 \%$ & & $2 \cdot 06(0 \cdot 62-4 \cdot 06)$ & $<0 \cdot 01,93 \cdot 8 \%$ & \\
\hline 2011-2014 & 76 & $48 \cdot 25(42 \cdot 68-53 \cdot 83)$ & $<0 \cdot 01,94 \cdot 9 \%$ & & $26 \cdot 02(20 \cdot 90-31 \cdot 47)$ & $<0 \cdot 01,95 \cdot 6 \%$ & & $1.96(0 \cdot 75-3 \cdot 57)$ & $<0 \cdot 01,91 \cdot 4 \%$ & \\
\hline Risk group\| & & & & $<0 \cdot 01$ & & & $0 \cdot 083$ & & & $<0 \cdot 01$ \\
\hline MSM & 121 & $51 \cdot 28(46 \cdot 15-56 \cdot 40)$ & $<0 \cdot 01,96 \cdot 1 \%$ & & $19 \cdot 98(16 \cdot 17-24 \cdot 07)$ & $<0 \cdot 01,95 \cdot 7 \%$ & & $0 \cdot 00(0 \cdot 00-0 \cdot 00)$ & $0 \cdot 577,0 \cdot 00 \%$ & \\
\hline HST & 76 & $38 \cdot 78(33 \cdot 08-44 \cdot 63)$ & $<0 \cdot 01,95 \cdot 4 \%$ & & $14 \cdot 88(10 \cdot 96-19 \cdot 23)$ & $<0 \cdot 01,95 \cdot 0 \%$ & & $9 \cdot 81(6 \cdot 44-13 \cdot 70)$ & $<0 \cdot 01,95 \cdot 3 \%$ & \\
\hline \multirow[t]{2}{*}{ Total } & 219 & $44 \cdot 54(40 \cdot 81-48 \cdot 30)$ & $<0 \cdot 01,96 \cdot 0 \%$ & & $16 \cdot 45(13 \cdot 82-19 \cdot 25)$ & $<0 \cdot 01,95 \cdot 5 \%$ & & $2 \cdot 55(1 \cdot 56-3 \cdot 73)$ & $<0 \cdot 01,93 \cdot 3 \%$ & \\
\hline & & \multicolumn{3}{|l|}{$\mathrm{B} / \mathrm{B}^{\prime}$} & \multicolumn{3}{|l|}{$\mathrm{C}$} & \multicolumn{3}{|l|}{ Others\# } \\
\hline Category & $N$ & $\begin{array}{l}\text { Proportion } \\
\%(95 \% \text { CI })\end{array}$ & $P^{*}, I^{2}$ & $P \dagger$ & $\begin{array}{l}\text { Proportion } \\
\%(95 \% \text { CI })\end{array}$ & $P^{*}, I^{2}$ & $P \dagger$ & $\begin{array}{l}\text { Proportion } \\
\%(95 \% \text { CI })\end{array}$ & $P^{*}, I^{2}$ & $P \dagger$ \\
\hline Regiont & & & & $<0.01$ & & & $0 \cdot 123$ & & & $0 \cdot 007$ \\
\hline Northeastern & 22 & $26 \cdot 15(16 \cdot 83-36 \cdot 60)$ & $<0 \cdot 01,93 \cdot 0 \%$ & & $0.00(0 \cdot 00-0 \cdot 06)$ & $0 \cdot 105,28 \cdot 5 \%$ & & $2 \cdot 40(1 \cdot 45-3 \cdot 51)$ & $<0 \cdot 01,77 \cdot 8 \%$ & \\
\hline Eastern & 115 & $24.99(19 \cdot 38-31 \cdot 03)$ & $<0 \cdot 01,97 \cdot 8 \%$ & & $0 \cdot 21(0 \cdot 03-0 \cdot 49)$ & $<0 \cdot 01,55 \cdot 8 \%$ & & $1 \cdot 17(0 \cdot 65-1 \cdot 78)$ & $<0 \cdot 01,73 \cdot 3 \%$ & \\
\hline Central & 27 & $28.90(15 \cdot 78-43.96)$ & $<0 \cdot 01,97 \cdot 4 \%$ & & $0.06(0 \cdot 00-0 \cdot 39)$ & $0 \cdot 363,6 \cdot 8 \%$ & & $3 \cdot 22(1 \cdot 39-5 \cdot 57)$ & $<0 \cdot 01,73 \cdot 8 \%$ & \\
\hline Northwestern & 7 & $5 \cdot 07(0 \cdot 64-12 \cdot 23)$ & $<0 \cdot 01,82 \cdot 4 \%$ & & $0.00(0 \cdot 00-0 \cdot 07)$ & $0.982,0.0 \%$ & & $0 \cdot 16(0 \cdot 00-1 \cdot 12)$ & $0 \cdot 082,46 \cdot 4 \%$ & \\
\hline Southwestern & 45 & $1 \cdot 82(1 \cdot 01-2 \cdot 81)$ & $<0 \cdot 01,57 \cdot 5 \%$ & & $1 \cdot 12(0 \cdot 01-3 \cdot 27)$ & $<0 \cdot 01,92 \cdot 6 \%$ & & $3 \cdot 33(1 \cdot 05-6 \cdot 50)$ & $<0 \cdot 01,93 \cdot 6 \%$ & \\
\hline Time period§ & & & & $<0.01$ & & & 0.768 & & & $0 \cdot 114$ \\
\hline Before 2000 & 9 & $52 \cdot 40(32 \cdot 41-72 \cdot 03)$ & $<0 \cdot 01,97 \cdot 2 \%$ & & $0 \cdot 10(0 \cdot 00-1 \cdot 89)$ & $<0 \cdot 01,73 \cdot 1 \%$ & & $1 \cdot 00(0 \cdot 00-4 \cdot 56)$ & $<0 \cdot 01,84 \cdot 3 \%$ & \\
\hline 2001-2004 & 14 & $44 \cdot 23(24 \cdot 99-64 \cdot 36)$ & $<0 \cdot 01,95 \cdot 6 \%$ & & $0.69(0.03-1.89)$ & $0 \cdot 045,42 \cdot 9 \%$ & & $0 \cdot 48(0 \cdot 00-2 \cdot 34)$ & $0 \cdot 007,54 \cdot 5 \%$ & \\
\hline $2005-2007$ & 34 & $34 \cdot 69(22 \cdot 05-48 \cdot 46)$ & $<0 \cdot 01,98 \cdot 2 \%$ & & $0.45(0 \cdot 01-1 \cdot 29)$ & $<0 \cdot 01,64 \cdot 7 \%$ & & $1 \cdot 65(0 \cdot 59-3 \cdot 06)$ & $<0 \cdot 01,73 \cdot 7 \%$ & \\
\hline 2008-2010 & 86 & $17 \cdot 77(12 \cdot 65-23 \cdot 47)$ & $<0 \cdot 01,96 \cdot 8 \%$ & & $0 \cdot 36(0 \cdot 01-1 \cdot 02)$ & $<0 \cdot 01,80 \cdot 0 \%$ & & $1 \cdot 46(0 \cdot 45-2 \cdot 86)$ & $<0 \cdot 01,90 \cdot 0 \%$ & \\
\hline 2011-2014 & 76 & $7 \cdot 17(4 \cdot 51-10 \cdot 32)$ & $<0 \cdot 01,94 \cdot 5 \%$ & & $0 \cdot 29(0 \cdot 00-0 \cdot 93)$ & $<0 \cdot 01,82 \cdot 0 \%$ & & $3 \cdot 35(2 \cdot 25-4 \cdot 62)$ & $<0 \cdot 01,80 \cdot 1 \%$ & \\
\hline
\end{tabular}




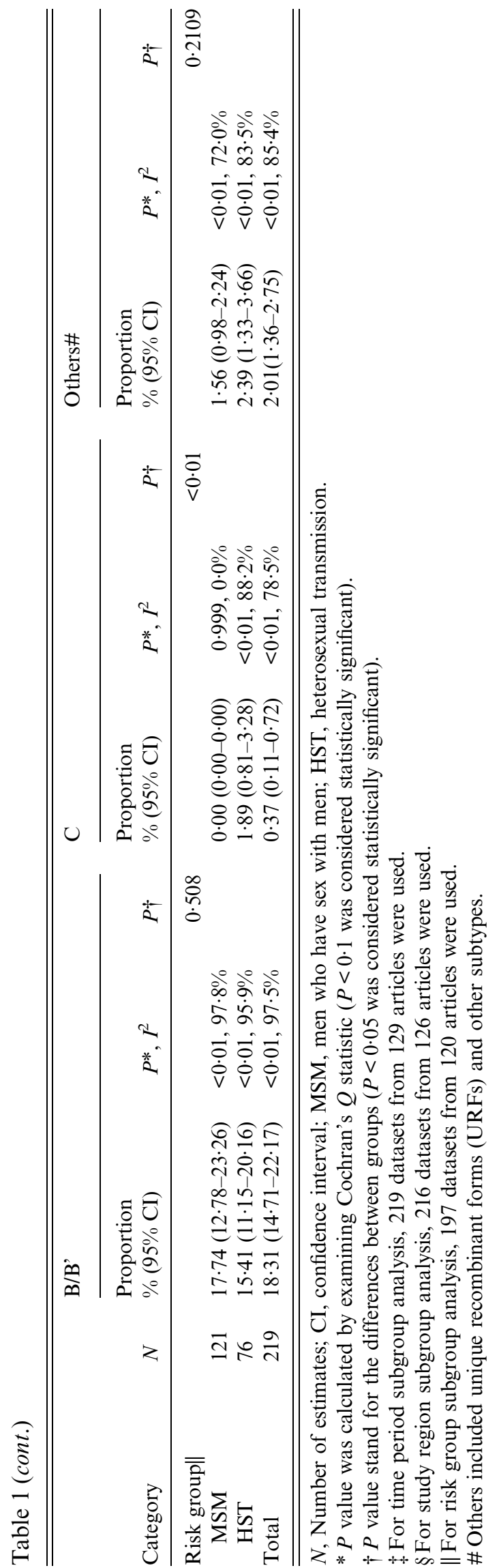

subgroups where relevant information was provided. Study time was further categorized roughly into five time periods to analyse the trends of different HIV-1 subtype distributions in sexual transmission. Figure 2 shows chronological trends of different HIV-1 subtypes in sexual transmission, MSM, and HST according to the midpoint of the year in which study participants were recruited. There was an upward trend in the proportion of CRF01_AE and CRF07_BC, especially among MSM. However, the proportion of subtype B/ B' showed a linearly downward trend. The proportion of CRF08_BC rose in the early stages and then decreased after about 2004, while it showed an upward trend in HST. Furthermore, CRF08_BC (9.81\%, 95\% CI 6.44-13.70) and subtype C $(1 \cdot 89 \%, 95 \%$ CI $0 \cdot 81-$ $3 \cdot 28$ ) mainly existed in HST (Table 1). The prevalence of subtype $\mathrm{C}$ and other subtypes in sexual transmission maintained a stable trend at a low level. Markedly, the proportion of URFs and other subtypes in MSM showed an upward trend.

Study location was also categorized roughly into five regions to further analyse the geographical distribution characteristics of HIV-1 subtypes. Figure 3 shows a diversity of HIV-1 subtype distributions in sexual transmission in different geographical regions. In the northeastern region, CRF01_AE $(57 \cdot 80 \%$, 95\% CI $45 \cdot 74-69 \cdot 44)$ and subtype B/B' (26.15\%, 95\% CI 16.83-36.60) were the predominant circulating strains. In the eastern region, CRF01_AE (48.95\%, 95\% CI 44.17-53.74), subtype B/B' (24.99\%, 95\% CI 19.38-31.03), and CRF07_BC (12.47\%, 95\% CI 9.84-15.35) were the predominant circulating strains. Furthermore, CRF01_AE (34.41\%, 95\% CI 24.75-44.72), subtype B/B' $(28 \cdot 90 \%, 95 \%$ CI 15.78-43.96), and CRF07_BC (19.52\%, 95\% CI 11.78-28.51) were also the three predominant circulating strains in the central region. In the northwestern region, HIV-1 prevalent strains were relatively simple, where CRF07_BC (65.89\%, 95\% CI 35.98-90.41) was the predominant circulating strain and CRF01_AE (22.68\%, 95\% CI 4.31-48.76) ranked second. In the southwestern region, CRF01_AE (36.16\%, 95\% CI 28.16-44.55), CRF07_BC (25.94\%, 95\% CI 18.20-34.46), and CRF08_BC (13.93\%, 95\% CI 7.93-21.13) were the three major circulating strains. Of note, URFs and other subtypes $(3 \cdot 33 \%, 95 \% \mathrm{CI}$ $1 \cdot 05-6 \cdot 50)$ were detected in significant proportions. This is the region where HIV-1 prevalent strains showed the highest diversity after the eastern and central regions.

In the different risk groups, the proportion of CRF01_AE, CRF07_BC, and subtype B/B' in MSM 

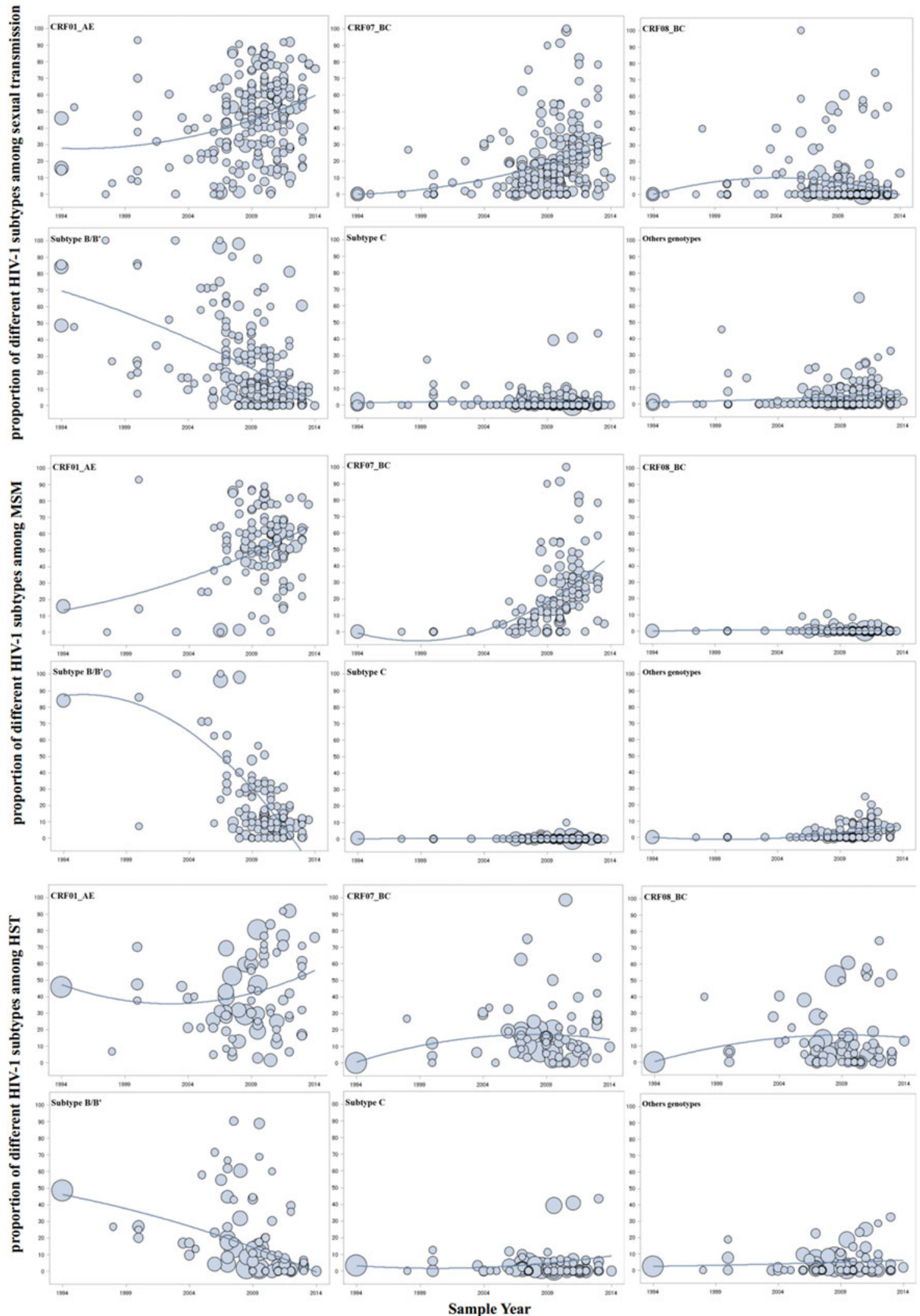

Fig. 2. Temporal trends in the yearly proportion of different HIV-1 subtypes in sexual transmission, men who have sex with men (MSM) and heterosexual transmission (HST) in China. The x-axis represents the midpoint of the year in which study samples were collected and analysed. The diameter of each bubble is proportional to the sample size of each study. The fitted line was plotted using a generalized linear mixed model regression. 


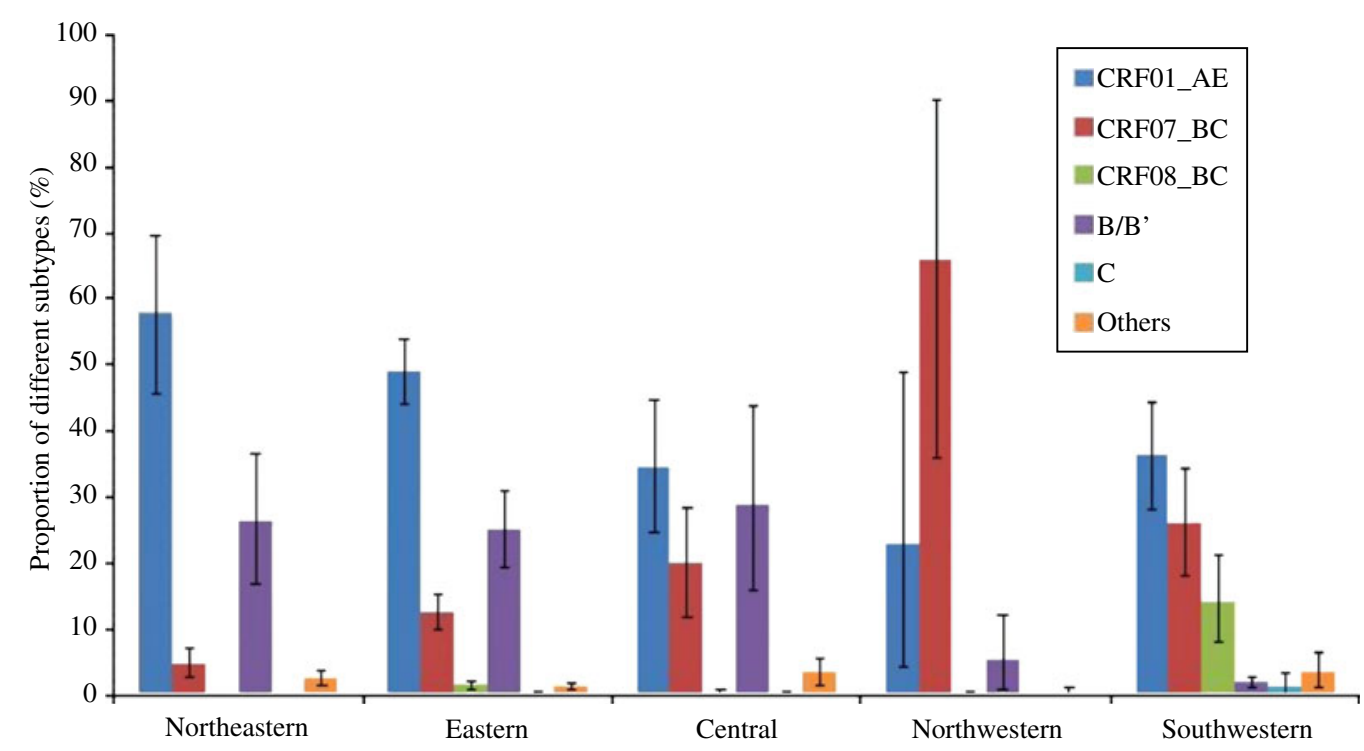

Fig. 3. Proportion of different HIV-1 subtypes in sexual transmission in different regions. The northeastern region includes Heilongjiang, Jilin and Liaoning provinces. The eastern region includes Beijing, Fujian, Guangdong, Hainan, Hebei, Hongkong, Jiangsu, Shandong, Shanghai, Taiwan, Tianjin, and Zhejiang provinces. The central region includes Anhui, Henan, Hubei, Hunan, Jiangxi, and Shanxi provinces. The northwestern region includes Gansu, Shaanxi, and Xinjiang provinces. The southwestern region includes Chongqing, Guangxi, Guizhou, Sichuan, and Yunnan, provinces.

were relatively higher than those in HST, while CRF08_BC and subtype C showed the opposite trend.

\section{DISCUSSION}

To our knowledge, this current meta-analysis is the largest to investigate the distribution of HIV-1 subtypes in sexual transmission in China [11, 18, 24]. The present study was also the first to provide a quantitative synthesis of the proportion of different HIV-1 subtype and subgroup analysis in sexual transmission in China with the use of proper statistical software. Through comprehensive searching of the literature, 130 eligible articles published from 2000 to 2015 and 18752 samples that were amplified successfully and genotyped were included in the analysis. The results showed that CRF01_AE, CRF07_BC, CRF08_BC, and subtype $\mathrm{B}$ were the four predominant subtypes in sexual transmission in China. The pooled proportion of different HIV-1 subtypes was similar to the national survey of HIV molecular epidemiology in China $[11,24]$. It partially verified that sexual transmission was the major pathway for the spread of HIV-1 in China [4, 25, 26].

The results showed that CRF01_AE represented the highest proportion both in MSM and HST. CRF01_AE, mainly through HST, was the only globally distributed non-B clade that originated in
Thailand but not Africa [27, 28]. It was first identified from female sex workers in northern Thailand in 1989 and later spread throughout Southeast Asia [29-31]. In China, CRF01_AE was originally detected in Yunnan Province in 1989 in intravenous drug users (IDUs) who were infected through commercial sex workers from Thailand [32]. It then rapidly spread across the country. Currently, there are seven distinct phylogenetic clusters of CRF01_AE in China, which mainly exist in heterosexuals, homosexuals, and IDUs [33]. Previous studies revealed that CRF01_AE strains with high frequencies of CXCR4 tropism were possibly related to the cause of a severe loss of CD4+ T cell counts and rapid disease progression [34, 35]. CRF01_AE had the highest proportion in different regions except for northwestern China. Moreover, it showed an upward trend, especially in MSM. Therefore, regular surveillance of HIV-1 subtypes is necessary to effectively prevent and control the HIV epidemic.

CRF07_BC and CRF08_BC, two major CRF strains in China, were first described in IDUs in Yunnan and Guangxi provinces [36, 37]. Through phylogenetic analysis, previous studies speculated that CRF07_BC possibly originated in China through recombination between subtype $B$ from Thailand and subtype C from India [38-40]. Phylogeographic analysis suggested CRF07_BC had two phylogenetic clusters. One originated from Xinjiang in the 1990s and 
spread to Liaoning, Jiangsu, Heilongiiang, Beijing and Guangdong through IDUs. Another one from Yunnan spread to Taiwan. However, CRF08_BC was first confirmed in Yunnan and later spread to Guangxi and Liaoning in the mid-1990s [41]. The proportion of CRF07/08_BC gradually increased in IDUs and later spread to other risk groups through crossinfection and transmission between IDUs and unprotected sexual behaviour [42, 43]. Our meta-analysis showed CRF07_BC in sexual transmission and CRF08_BC in HST were increasing, due to a high correlation between unprotected sexual intercourse and drug use increasing HIV transmission in China [42-44]. Thus, novel and effective prevention strategies are necessary for drug use in unprotected sexual behaviours.

Our results indicated that the proportion of subtype $\mathrm{B}$ rapidly decreased, especially in MSM. This is consistent with other studies where CRF01_AE became the predominant circulating strain, replacing subtype B, in MSM [16, 45]. In China, subtype B has four distinct subgroups, namely B (Thai-B), BJ-B (Beijing-B), Pan-B (Pandemic-B), and TW-B (Taiwan-B) [46]. Thai-B was transmitted from IDUs in Yunnan to former plasma donors (FPDs) in central China in the 1990s. Then it rapidly spread across the country through sexual transmission [11, 47, 48]. However, BJ-B, TW-B, and Pan-B (circulating in the United States and Europe) lineages were restricted to particular regions. Although subtype B showed a rapid downward trend in sexual transmission, it still represented a large proportion. Furthermore, the recombinant virus of subtype B and other subtypes were constantly emerging in China, such as CRF_01B, CRF_BC, CRF65_cpx, etc. [7, 49]. These results significantly impact HIV therapeutics and vaccine development in China.

Geographical sub-analysis showed the diverse distribution of HIV-1 subtypes in different regions. The distribution of HIV-1 subtypes was relatively simple in the northeastern and northwestern regions, comparatively diverse in the eastern and central regions, with the highest diversity in the southwestern region. This is relevant to the differences in the drivers of HIV-1 transmission. In the northeast, CRF01_AE and subtype $\mathrm{B}$ became the two predominant circulating strains owing to heterosexuals and MSM dominating HIV-1 transmission [11, 50]. In the east, there were multiple risk groups due to the booming economy attracting large numbers of migrants from all over the country. The coexistence of multiple risk groups lead to a diversity of the HIV-1 virus [49, 51, 52]. In the central region, FPD dominated the spread of HIV-1 in the early stage, and later was gradually replaced by sexual transmission [4, 53]. Furthermore, the central and eastern regions have maintained close contacts due to their adjacent location and economic exchanges. Therefore, the distributions of HIV-1 subtypes were similar in these two regions. In the northwestern region, the overall prevalence of CRF07_BC was higher mainly due to it originating from IDUs in Xinjiang [41]. The proportion of CRF01_AE, higher than in previous studies [11], suggested that sexual transmission was shifting the major pathway of the spread of HIV-1 in this region. The southwestern region included Yunnan, Guizhou, Guangxi and Sichuan provinces, which was the region worst affected by HIV due to cross-infection from different strains and spread in multiple risk groups. In particular, Yunnan was the first location where the HIV epidemic was identified in China [54]. Furthermore, previous studies revealed that almost all of the HIV-1 subtypes from other regions originated in Yunnan [54-56]. Our results also suggested that HIV-1 prevalent strains had the highest diversity in this region. The diverse distribution of HIV-1 subtypes poses a significant challenge to disease control and surveillance in the different regions of China.

Additionally, our meta-analysis found the proportions of CRF01_AE, CRF07_BC, and subtype B in MSM were relatively higher than those in HST, while CRF08_BC and subtype C showed the opposite trend. Additionally, the prevalence of CRF08_BC was restricted to the southwestern region. Subtype $C$ and other subtypes in sexual transmission were consistently low. However, the proportion of URFs and other subtypes in MSM showed an upward trend. These results provide important information to effectively guide the prevention and control of HIV-1 in China.

Our study also faced several important limitations. First, significant heterogeneity was observed and in the subgroup analysis. It might be attributed to the small sample size of some of the studies included in this analysis, or that a high diversity of HIV-1 subtype distribution existed in different regions, time periods, and risk groups. Second, as most studies included in the analysis did not provide detailed categorical data on subtypes B and B', we merged and analysed them together. Finally, even though the funnel plot and Egger's test suggests an overall low risk of publication bias, we cannot avoid the other biases, such as language bias, database bias, multiple publication bias, etc. 
Despite these limitations, the present study represents the first comprehensive investigation of HIV-1 subtypes in sexual transmission in China. These HIV-1 prevalent strains have evolved into complicated subtypes, and major subtypes include CRF01_AE, CRF07_BC, CRF08_BC, and subtype B. In addition, the proportion of different HIV-1 subtypes has changed constantly over time. New recombinant strains are also constantly emerging in China. The high genetic variability of HIV-1 poses a significant challenge for disease diagnosis, treatment, and vaccine development in China.

\section{SUPPLEMENTARY MATERIAL}

For supplementary material accompanying this paper visit http://dx.doi.org/10.1017/S0950268816000212.

\section{ACKNOWLEDGEMENTS}

This work was supported by the Science and Technology Project in Wuxi City (Project No. CSE01N1232), and the Doctoral Program Foundation of Institutions of Higher Education of China (Project No. 20130092110048).

\section{DECLARATION INTEREST}

None.

\section{REFERENCES}

1. Hao Y, et al. A retrospective study of HIV/AIDS situation: a ten-year implementation of 'four frees and one care policy' in China. Chinese Journal of Disease Control \& Prevention 2014; 18: 369-374.

2. Ministry of Health, People's Republic of China, Joint United Nations Programme on HIV/AIDS, World Health Organization. 2011 Estimates for the HIV/ AIDS epidemic in China. Beijing, China, 2011.

3. NCAIDS/STD, Chinese CDC. Update on the AIDS/ STD epidemic in China and main response in control and prevention in July, 2015. Chinese Journal of AIDS and STD 2015; 21: 749.

4. National Health and Family Planning Commission of the People's Republic of China. 2014 China AIDS Response Progress Report. Beijing, China, 2014.

5. Hemelaar J. The origin and diversity of the HIV-1 pandemic. Trends in Molecular Medicine 2012; 18: 182-192.

6. Lau KA, Wong JJ. Current trends of HIV recombination worldwide. Infectious Disease Reports 2013; 5 (Suppl. 1): e4.

7. HIV sequence database. HIV circulating recombinant forms (CRFs) (http://www.hiv.lanl.gov/content/sequence/HIV/CRFs/CRFs.html). Accessed 15 July 2015.

8. Hemelaar J. Implications of HIV diversity for the HIV-1 pandemic. Journal of Infection 2013; 66: 391-400.
9. Shao Y. HIV/AIDS: perspective on China. AIDS Patient Care and STDs 2001; 15: 431-432.

10. Group for HIV Molecular Epidemiologic Survey. Project Summary for National HIV Molecular Epidemiology and Database Establishment. National Public Welfare Research Fund, Ministry of Science and Technology of China, 2004.

11. He X, et al. A comprehensive mapping of HIV-1 genotypes in various risk groups and regions across China based on a nationwide molecular epidemiologic survey. PLOS ONE 2012; 7: e47289.

12. Hue $\mathbf{S}$, et al. HIV-1 pol gene variation is sufficient for reconstruction of transmissions in the era of antiretroviral therapy. AIDS 2004; 18: 719-728.

13. Walter BL, et al. Functional characteristics of HIV-1 subtype C compatible with increased heterosexual transmissibility. AIDS 2009; 23: 1047-1057.

14. Bhargava M, et al. Do HIV-1 non-B subtypes differentially impact resistance mutations and clinical disease progression in treated populations? Evidence from a systematic review. Journal of the International AIDS Society 2014; 17: 18944.

15. Sui HS, et al. Different frequencies of drug resistance mutations among HIV-1 subtypes circulating in China: a comprehensive study. PLoS ONE 2014; 9.

16. Wang W, et al. The dynamic face of HIV-1 subtypes among men who have sex with men in Beijing, China. Current HIV Research 2011; 9: 136-139.

17. Liberati A, et al. The PRISMA statement for reporting systematic reviews and meta-analyses of studies that evaluate health care interventions: explanation and elaboration. Journal of Clinical Epidemiology 2009; 62: e1-34.

18. Krogstad P, et al. Mother-to-child transmission in the United States of subtypes D and A/G human immunodeficiency virus type 1. AIDS Research and Human Retroviruses 2002; 18: 413-417.

19. Zhang L, et al. Prevalence of HIV-1 subtypes among men who have sex with men in China: a systematic review. International journal of STD \& AIDS 2015; 26: 291-305.

20. Liu $\mathbf{H}$, et al. Emerging trends of HIV drug resistance in Chinese HIV-infected patients receiving first-line highly active antiretroviral therapy: a systematic review and meta-analysis. Clinical Infectious Diseases 2014; 59: 1495-1502.

21. Higgins JP, Thompson SG. Quantifying heterogeneity in a meta-analysis. Statistics in Medicine 2002; 21: 1539-1558.

22. Huedo-Medina TB, et al. Assessing heterogeneity in meta-analysis: $Q$ statistic or $I^{2}$ index? Psychological Methods 2006; 11: 193-206.

23. R Core Team (2013). R: A language and environment for statistical computing. R Foundation for Statistical Computing, Vienna, Austria (http://www.R-project. org/). Accessed 5 July 2015.

24. Su YY, et al. Distribution of HIV-1 genotypes in China: a systematic review. Chinese Journal of Epidemiology 2014; 35: 1164-1168.

25. Chow EP, et al. Behavioral interventions improve condom use and HIV testing uptake among female sex 
workers in china: a systematic review and meta-analysis. AIDS Patient Care STDS 2015; 29: 454-60.

26. Lu L, et al. The changing face of HIV in China. Nature 2008; 455: 609-611.

27. Gao F, et al. The heterosexual human immunodeficiency virus type 1 epidemic in Thailand is caused by an intersubtype (A/E) recombinant of African origin. Journal of virology 1996; 70: 7013-7029.

28. Angelis K, et al. Global dispersal pattern of hiv type 1 subtype CRF01_AE: a genetic trace of human mobility related to heterosexual sexual activities centralized in Southeast Asia. Journal of Infectious Diseases 2015; 211: 1735-1744.

29. Carr JK, et al. Full-length sequence and mosaic structure of a human immunodeficiency virus type 1 isolate from Thailand. Journal of Virology 1996; 70: 5935-5943.

30. Nelson KE, et al. Risk factors for HIV infection among young adult men in northern Thailand. Journal of the American Medical Association 1993; 270: 955-960.

31. Hemelaar J, et al. Global trends in molecular epidemiology of HIV-1 during 2000-2007. AIDS 2011; 25: 679-689.

32. Zheng $\mathbf{X}$, et al. Injecting drug use and HIV infection in southwest China. AIDS 1994; 8: 1141-1147.

33. Feng Y, et al. The rapidly expanding CRF01_AE epidemic in China is driven by multiple lineages of HIV-1 viruses introduced in the 1990s. AIDS 2013; 27: 1793-1802.

34. Li Y, et al. CRF01_AE subtype is associated with X4 tropism and fast HIV progression in Chinese patients infected through sexual transmission. AIDS 2014; 28: 521-530.

35. Li X, et al. Evidence that HIV-1 CRF01_AE is associated with low CD4+T cell count and CXCR4 coreceptor usage in recently infected young men who have sex with men (MSM) in Shanghai, China. PLoS ONE 2014; 9: e89462.

36. Su L, et al. Characterization of a virtually full-length human immunodeficiency virus type 1 genome of a prevalent intersubtype (C/B') recombinant strain in China. Journal of Virology 2000; 74: 11367-11376.

37. Piyasirisilp S, et al. A recent outbreak of human immunodeficiency virus type 1 infection in southern China was initiated by two highly homogeneous, geographically separated strains, circulating recombinant form $\mathrm{AE}$ and a novel BC recombinant. Journal of Virology 2000; 74: 11286-11295.

38. Ye JR, et al. Molecular epidemiological characteristics of HIV-1 B'/C strains in Beijing. National Medical Journal of China 2013; 93: 2301-2304.

39. Luo CC, et al. HIV-1 subtype C in China. Lancet 1995; 345: 1051-1052.

40. Graf M, et al. Cloning and characterization of a virtually full-length HIV type 1 genome from a subtype B'-Thai strain representing the most prevalent B-clade isolate in China. AIDS Research and Human Retroviruses 1998; 14: 285-288.
41. Liu J, Zhang C. Phylogeographic analyses reveal a crucial role of Xinjiang in HIV-1 CRF07_BC and HCV 3a transmissions in Asia. PLoS ONE 2011; 6: e23347.

42. Tee KK, et al. Temporal and spatial dynamics of human immunodeficiency virus type 1 circulating recombinant forms 08_BC and 07_BC in Asia. Journal of Virology 2008; 82: 9206-9215.

43. Chen YJ, et al. Molecular Epidemiology of HIV-1 Infection in Taiwan from 2005 to 2008: further spread of CRF07_BC and Emergence of CRF07_BC/Subtype B dual infection. Journal of Acquired Immune Deficiency Syndromes 2012; 59: 438-446.

44. Chen X, et al. Club drugs and HIV/STD infection: an exploratory analysis among men who have sex with men in Changsha, China. PLoS ONE 2015; 10: e0126320.

45. Chen JHK, et al. Molecular epidemiological study of HIV-1 CRF01_AE transmission in Hong Kong. Journal of Acquired Immune Deficiency Syndromes 2009; 51: 530-535.

46. Ye JR, et al. Phylogenetic and temporal dynamics of human immunodeficiency virus type 1B in China: four types of B strains circulate in China. AIDS Research and Human Retroviruses 2014; 30: 920-926.

47. Li L, et al. HIV-1 Thai B strain has spread out of former plasma donors into general population through sexual contact in Henan, China. Journal of Medical Virology 2016; 88: 614-621.

48. Li Z, et al. Tracing the origin and history of HIV-1 subtype B' epidemic by near full-length genome analyses. AIDS (London, England) 2012; 26: 877-884.

49. Chen JH, et al. Increased genetic diversity of HIV-1 circulating in Hong Kong. PLoS ONE 2010; 5: e12198.

50. Dai D, et al. The biological characteristics of predominant strains of HIV-1 genotype: modeling of HIV-1 infection among men who have sex with men. Journal of Medical Virology 2015; 87: 557-568.

51. Chen S, et al. Molecular epidemiology of human immunodeficiency virus type 1 in Guangdong province of southern China. PLoS ONE 2012; 7: e48747.

52. Yao X, et al. Rising epidemic of HIV-1 infections among general populations in Fujian, China. Journal of Acquired Immune Deficiency Syndromes 2012; 60: 328-335.

53. Yang RR, et al. The comparison of human immunodeficiency virus type 1 transmission between couples through blood or sex in central China. Japanese Journal of Infectious Diseases 2010; 63: 283-285.

54. Lu L, et al. The changing face of HIV in China. Nature 2008; 455: 609-611.

55. Zhang Y, et al. Dominance of HIV-1 subtype CRF01_AE in sexually acquired cases leads to a new epidemic in Yunnan Province of China. PLoS Medicine 2006; 3: 2065-2077.

56. Chen M, et al. Emerging variability in HIV-1 genetics among recently infected individuals in Yunnan, China. PLoS ONE 2013; 8: e60101. 